\title{
Astrophysical Applications of the Iron Project: Ionization Structure and Spectra of Iron in Nebulae
}

\author{
Manuel A. Bautista ${ }^{1}$ and Anil K. Pradhan ${ }^{1}$ \\ ${ }^{1}$ Department of Astronomy, The Ohio State University, Columbus, Ohio 43210-1106, U.S.A.
}

We study the physical structure of H II regions, including Planetary Nebulae (PNe), through detailed analysis of the ionization structure and spectra of iron in relevant ionization stages. This results from our work in the Ohio State group on: (i) calculation of new and improved atomic data for Fe I-VI (photoionization cross sections, recombination rates, collision strengths, and transition probabilities); (ii) detailed study of ionization, recombination, and excitation mechanisms and radiative transfer effects under nebular conditions; (iii) study of the physical structure and kinematics of nebulae and their ionization fronts. This includes further examination of high density regions deduced from the spectroscopic analysis of optical [Fe II], [Ni II], and [O I] emission (Bautista etal 1996; and references therein). The study also addresses the issue of chemical abundances in fully ionized and partially ionized zones of nebulae.

The new atomic data are much more accurate and extensive than in previous calculations and the differences are considerable. For instance, the new photoionization cross sections differ from previous values by up to three orders of magnitude for $\mathrm{Fe} \mathrm{I}$ and about an order of magnitude for Fe II, Fe IV and Fe V. Consequently, the new recombination rate coefficients are also different from previous results by up to a factor of four from previous data at typical nebular temperatures. The new collisional excitation rates and transition probabilities also differ significantly from earlier calculations.

By using the new atomic data the calculated ionization structure of $\mathrm{Fe}$ in nebulae is altered significantly with respect to previous models. The emission spectra of $\mathrm{Fe}$ is calculated for several models considering a variety of excitation mechanisms, i.e. collisional excitation, fluorescence, and excitation by recombination into excited states. It is found that a high density, partially ionized zone at the ionization front should exist in most $\mathbf{H}$ II regions if the observed optical [Fe II], [Ni II], and [O I] emission is to be reproduced by photoionization modeling. This agrees with spectral diagnostics of several objects which indicate that optical [Fe II] lines are collisional excited in regions with high electron densities.

\section{REFERENCES}

Bautista, M. A., Peng, J., \& Pradhan, A. K. 1996, Ap. J. 460, 372 